\title{
CRIBADO DE DISTRÉS EMOCIONAL EN POBLACIÓN ONCOLÓGICA ADULTA: VALIDACIÓN DE INSTRUMENTOS
}

\author{
Autor: Paula Martínez López \\ Tipo de trabajo: Tesis Doctoral por compilación de publicaciones \\ Directores: Dra. Yolanda Andreu Vaillo y Dra. María José Galdón Garrido \\ Centro: Departamento de Personalidad, Evaluación y Tratamientos Psicológicos. \\ Facultad de Psicología. Universidad de Valencia. \\ Fecha de lectura: 25 de enero de 2016. \\ E-mail: paula.martinez@uv.es \\ Estudio financiado por el Ministerio de Economía y Competitividad.
}

La investigación ha demostrado la presencia de niveles de distrés (o malestar emocional) clínicamente significativos en aproximadamente tres de cada diez pacientes oncológicos (Mitchell et al., 2011). Sin embargo, en la actualidad, menos de la mitad de éstos pacientes son identificados y derivados a los servicios de asistencia psicosocial correspondientes (NCCN, 2015). El fracaso a la hora de detectar y tratar adecuadamente sus necesidades emocionales conlleva una serie de importantes consecuencias negativas que repercuten no solo en la calidad de vida de los pacientes (McDowell et al., 2010) y en su nivel de satisfacción con la atención médica recibida sino también en los gastos de la asistencia sanitaria (Bultz y Holland, 2006; Carlson y Bultz, 2004; Kroenke et al., 2010). Ante esta problemática, distintas organizaciones profesionales y organismos internacionales han reclamado la necesidad de incluir la detección y el control del distrés emocional como una parte esencial de la atención integral al paciente oncológico. $\mathrm{Al}$ respecto, cabe destacar la declaración en 2004 del distrés emocional como el " 6 a signo vital" por la agencia de salud pública del Gobierno Federal de Canadá -Canadian Strategy for Cancer Control-. Una propuesta que, actualmente, es respaldada por más de setenta organismos internacionales y organizaciones profesionales. Entre las actuaciones más significativas con respecto al tema, se incluye el desarrollo de estándares y directrices de práctica clínica para la detección y tratamiento del distrés en población oncológica. En ellos, se insiste en la necesidad de detectar y controlar rutinariamente los niveles de distrés, así como las causas que lo originan. Dentro de este contexto, el desarrollo y validación de instrumentos estandarizados de cribado que permitan identificar a aquellos pacientes que experimentan niveles elevados de distrés se convierte en una meta primordial de la investigación en psico-oncología. La presente tesis, por compilación de publicaciones, aporta datos sobre la adaptación y validación de un grupo de instrumentos de evaluación para su uso como herramientas de cribado del distrés emocional en población oncológica adulta española. En concreto, la primera 
publicación ( $N=892)$ informa sobre la adecuación de utilizar la puntuación total de la Escala Hospitalaria de Ansiedad y Depresión -HADS- (Zigmond y Snaith, 1983) como medida general de distrés y dota al instrumento de un punto de corte óptimo para la selección, en población oncológica, de casos clínicamente significativos de distrés. Asimismo, la segunda publicación ( $N=962)$ analiza la precisión diagnóstica del Termómetro de Distrés -DT- (Roth et al., 1998) y evidencia el comportamiento satisfactorio de dicho instrumento para el cribado rutinario del distrés, esto es, para descartar "no casos" con un número reducido de falsos negativos; sin embargo, se señala su moderada capacidad para la detección de "caso", dado que acumula un número considerable de falsos positivos. Este trabajo, además, ofrece datos sobre prevalencia de distrés clínico en la muestra de estudio e informa sobre la existencia de una discrepancia entre los niveles de distrés identificados y el conocimiento/utilización de los recursos psicosociales existentes. La tercera publicación $(N=385)$ estudia una posible mejora de la precisión diagnóstica del DT -en particular, del nivel de especificidad- por su combinación con el Termómetro de Impacto -IT(Akizuki et al., 2005). En definitiva, las diferentes publicaciones que recoge la tesis doctoral tratan de aportar datos que nos permitan dar respuesta a cuestiones básicas y de actualidad relacionadas con la capacidad o idoneidad de determinados instrumentos para evaluar distrés emocional en la población oncológica adulta española; así como dotar a los profesionales sanitarios de instrumentos breves, validados y útiles para abordar esta tarea en la práctica clínica. 